Pacific Journal of Mathematic 


\section{TOTALLY GEODESIC HYPERSURFACES OF KAEHLER MANIFOLDS}

\section{SAMUel I. GoldBerG}

It is known that a $C^{\infty}$ orientable totally umbilical hypersurface $P$ with nonzero mean curvature of a Kaehler manifold $M$ is a normal contact manifold. Moreover, if $M=C_{n}$ with the flat Kaehler metric, $P$ can be realized as a normal contact metric manifold of positive constant curvature. It is the main purpose of this paper to obtain corresponding results for cosymplectic manifolds.

The direct product of two normal almost contact manifolds can be endowed with a complex structure. For cosymplectic manifolds more is obtained. Indeed, the direct product of two cosymplectic manifolds can be given a Kaehlerian structure. This is particularly true of orientable totally geodesic hypersurfaces of a Kaehler manifold.

Our notion of a cosymplectic manifold differs from the one given by $P$. Libermann in [3] and was given by D. Blair [1].

THeOREM 1. A necessary and sufficient condition that a $C^{\infty}$ orientable hypersurface $P$ of a Kaehler manifold $M$ be cosymplectic with almost contact form $\eta$ is that its second fundamental form $H$ be proportional to $\eta \otimes \eta$, that is

$$
H=h \eta \otimes \eta,
$$

where $h=H(\xi, \xi)$, the vector field $\xi$ being the contravariant form of $\eta$ with respect to the almost contact metric.

CoROLLARY 1. A $C^{\infty}$ orientable totally geodesic hypersurface of a Kaehler manifold is a cosymplectic manifold.

A corresponding result was obtained by Y. Tashiro [6] for totally umbilical hypersurfaces.

For complete simply connected cosymplectic manifolds an application of the de Rham decomposition theorem (see [2]) yields

CoROLlary 2. $A C^{\infty}$ complete simply connected orientable totally geodesic hypersurface of a Kaehler manifold is a product with one factor Kaehlerian.

THEOREM 2. A cosymplectic hypersurface of $C_{n}$ with the flat Kaehler metric is locally flat. 
For the corresponding statement concerning normal contact hypersurfaces the reader is referred to [6].

A. Morimoto [4] has shown that the direct product of two normal almost contact manifolds can be given a complex structure. For cosymplectic manifolds we obtain more.

THEOREM 3. The direct product of two cosymplectic manifolds can be given a Kaehlerian structure.

\section{Applying Corollary 1 we obtain}

CoRollary 3. The direct product of two $C^{\infty}$ orientable totally geodesic hypersurfaces of a Kaehler manifold is a Kaehler manifold.

2. Almost contact manifolds. An almost contact structure $(\phi, \xi, \eta)$ on a $(2 n+1)$-dimensional $C^{\infty}$ manifold $P$ is given by a tensor field $\phi$ of type $(1,1)$, a vector field $\xi$ and a 1 -form $\eta$ on $P$ called the contact form such that

$$
\begin{gathered}
\eta(\xi)=1, \\
\phi(\xi)=0, \quad \eta \circ \phi=0, \\
\phi^{2}=-I+\eta(\cdot) \xi,
\end{gathered}
$$

where $I$ is the identity transformation field. If $P$ has a $(\dot{\phi}, \xi, \eta)$ structure then we can find a Riemann metric $($, ) such that

$$
\begin{aligned}
\eta & =(\xi, \cdot), \\
(\phi X, \phi Y) & =(X, Y)-\eta(X) \eta(Y),
\end{aligned}
$$

so that $\phi$ is skew-symmetric with respect to ( , ). $P$ is then said to have a $(\phi, \xi, \eta,()$,$) -structure.$

The almost contact structure is called normal if for any vector fields $X, Y$ on $P$

$$
[X, Y]+\phi[\phi X, Y]+\phi[X, \phi Y]-[\phi X, \phi Y]=d \eta(X, Y) \xi .
$$

A $(2 n+1)$-dimensional $C^{\infty}$ manifold is said to have a contact structure, and is then called a contact manifold, if it carries a global 1-form $\eta$ such that

$$
\eta \wedge(d \eta)^{n} \neq 0
$$

It can be shown that there exists a $(\phi, \xi, \eta,()$,$) -structure on a con-$ tact manifold $P$ such that 


$$
d \eta=(\phi X, Y)
$$

$P$ is then called a contact metric manifold.

An almost contact metric structure $(\phi, \xi, \eta,()$,$) is called quasi-$ Sasakian if it is normal and its fundamental form $\Phi$, where $\Phi(X, Y)=$ $(\phi X, Y)$, is closed. The quasi-Sasakian manifolds may be classified according to the rank of $\eta$. The 1-form $\eta$ has rank $2 p$ if $(d \eta)^{p} \neq 0$ and $\eta \wedge(d \eta)^{p}=0$, and has rank $2 p+1$ if $\eta \wedge(d \eta)^{p} \neq 0$ and $(d \eta)^{p+1}=0$. There are no quasi-Sasakian structures of even rank [1]. If the rank is maximal, the almost contact manifold is a Sasakian manifold, and if the rank is 1 , it is a cosymplectic manifold.

3. Almost contact hypersurfaces. Let $M$ be an almost hermitian manifold of real dimension $2 n$ with almost complex structure tensor $J$. Then, in terms of the hermitian metric $\langle$,$\rangle of M$

$$
\langle J x, J y\rangle=\langle x, y\rangle
$$

for every pair of tangent vectors $x, y \in M_{m}$-the tangent space at $m \in M$. If $P$ is a smooth orientable hypersurface imbedded in $M$ with imbedding $i: P \rightarrow M$, the induced metric on $P$ is defined in terms of the metric on $M$ by

$$
(x, y)=\left\langle i_{*} x, i_{*} y\right\rangle
$$

for each pair of tangent vectors $x, y \in P_{m}$.

A Riemannian connexion $D$ on a Riemannian manifold with metric $\langle$,$\rangle is characterized by the properties:$

$$
\begin{gathered}
D_{X} Y-D_{Y} X=[X, Y] \text { and } \\
Z\langle X, Y\rangle=\left\langle D_{Z} X, Y\right\rangle+\left\langle X, D_{Z} Y\right\rangle .
\end{gathered}
$$

Let $N_{m}$ be the unit normal of $P$ at $m$ with orientation determined by that of $P$. Let $\nabla$ be the Riemannian connexion on $M$. The Weingarten $\operatorname{map} W: P_{m} \rightarrow P_{m}$ is given by

$$
W(x)=\nabla_{x} N_{m}, \quad x \in P_{m} .
$$

(We write $x$ for $i_{*} x$ in the sequel, with no resulting confusion, in order to simplify our notation.) The second fundamental form $H: P_{m} \times P_{m} \rightarrow R$ of $P$ is the symmetric bilinear form

$$
H(x, y)=(W x, y) \text {. }
$$

If $D$ is the induced Riemannian connexion on $P$, then

$$
D_{x} Y=\nabla_{x} Y+H(x, y) N_{m},
$$

where $x \in P_{m}$ and $\vdots Y$ is a vector field on $P$ extending $y$. Set 


$$
\eta(x)=\langle J x, N\rangle, \quad x \in P_{m}
$$

and

$$
\Phi(x, y)=\langle J x, y\rangle, \quad x, y \in P_{m} .
$$

Formula (3.1) says that $\Phi$ is skew-symmetric with respect to $\langle$,$\rangle .$ If $\xi=(\eta$,$) is the contravariant form of \eta$ with respect to $($,$) , then$ $\xi$ is a vector field on $P$ with the property

$$
J \xi=N \text {. }
$$

Thus,

$$
\begin{aligned}
(\iota(\xi) \Phi)(X) & =\Phi(\xi, X) \\
& =\langle J \xi, X\rangle \text { by }(3.7) \\
& =\langle N, X\rangle \text { by }(3.8) \\
& =0,
\end{aligned}
$$

where $X \in E(P)$, the module of vector fields on $P$.

Now, in terms of $\Phi$ and (, ) an endomorphism $\phi$ of $E(P)$ is defined by the equation

$$
(\dot{\phi} X, Y)=\Phi(X, Y), \quad X, Y \in E(P) .
$$

Since $\Phi$ is skew-symmetric

$$
(\phi X, Y)=-(X, \phi Y) \text {. }
$$

Moreover,

$$
\phi X=J X-\eta(X) N, \quad X \in E(P) .
$$

It follows that

$$
(\phi X, \phi Y)=(X, Y)-\eta(X) \eta(Y) .
$$

For, by (3.10), (3.7), (3.12) and (3.6), $(\phi X, \phi Y)=\Phi(X, \phi Y)=\langle J X, \phi Y\rangle=$ $\langle J X, J Y-\eta(Y) N\rangle=\langle J X, J Y\rangle-\eta(Y)\langle J X, N\rangle=\langle X, Y\rangle-\eta(Y) \eta(X)$. In addition, since $\left(\phi^{2} X, Y\right)=-(\phi X, \phi Y)=\eta(X) \eta(Y)-(X, Y)$

$$
\phi^{2} X=-X+\eta(X) \xi \text {. }
$$

Applying (3.9) and (3.10), we obtain

$$
\phi \xi=0 .
$$

Thus, from (3.11) and (3.13)

$$
\eta \circ \phi=0 .
$$


We conclude that, $P$ has a $(\phi, \xi, \eta,()$,$) -structure.$

Proposition 1. A $C^{\infty}$ orientable hypersurface $P$ of an almost hermitian manifold $M$ has a naturally induced almost contact structure.

If the fundamental 2-form $\Omega$ of $M$ where $\Omega(X, Y)=\langle J X, Y\rangle$ is closed (that is, if $M$ is an almost Kaehler manifold) then $\Phi$ is closed. Indeed, $\Phi=i^{*} \Omega$ directly from the definitions of $\Phi$ and $\Omega$.

We compute $D_{X} \Phi$ :

$$
\begin{aligned}
\left(D_{X} \Phi\right)(Y, Z)= & X \Phi(Y, Z)-\Phi\left(D_{X} Y, Z\right)-\Phi\left(Y, D_{X} Z\right) \\
= & X(\phi Y, Z)+\left(\phi Z, D_{X} Y\right)-\left(\phi Y, D_{X} Z\right) \\
= & X(\phi Y, Z)+\left\langle\phi Z, \nabla_{X} Y\right\rangle-\left\langle\phi Y, \nabla_{X} Z\right) \\
= & X\langle J Y, Z\rangle+\left\langle J Z, \nabla_{X} Y\right\rangle-\left\langle J Y, \nabla_{X} Z\right\rangle \\
& -\left\langle\eta(Z) N, \nabla_{X} Y\right\rangle+\left\langle\eta(Y) N, \nabla_{X} Z\right\rangle \\
= & \left(\nabla_{X} Q\right)(Y, Z)-\eta(Z)\left\langle N, \nabla_{X} Y\right\rangle+\eta(Y)\left\langle N, \nabla_{X} Z\right\rangle \\
= & \left(\nabla_{X} \Omega\right)(Y, Z)+\eta(Z) H(X, Y)-\eta(Y) H(X, Z)
\end{aligned}
$$

for all $X, Y, Z \in E(P)$.

If $M$ is Kaehlerian, $\nabla_{X} \Omega=0$, so

$$
\left(D_{X} \Phi\right)(Y, Z)=\eta(Z) H(X, Y)-\eta(Y) H(X, Z) \text {. }
$$

Moreover,

$$
\left(D_{X} \eta\right)(Y)=H(X, \phi Y)
$$

For,

$$
\begin{aligned}
\left(D_{X} \eta\right)(Y) & =\left(Y, D_{X} \xi\right) \\
& =\left\langle Y, \nabla_{X} \xi\right\rangle \text { by }(3.5) \\
& =\left\langle J Y, J \nabla_{X} \xi\right\rangle \text { by }(3.1) \\
& =\left\langle J Y, \nabla_{X} J \xi\right\rangle \text { since } \nabla_{X} \Omega \text { vanishes } \\
& =\left\langle J Y, \nabla_{X} N\right\rangle \text { by }(3.8) \\
& =(\phi Y, W X) \text { by }(3.3) \\
& =(W \phi Y, X) \text { since } W \text { is self-adjoint } \\
& =H(X, \phi Y) \text { by }(3.4) .
\end{aligned}
$$

4. Proof of Theorem 1. If $H=h \eta \otimes \eta$, then by (3.17), $D_{X} \Phi=0$, and by (3.18), $\left(D_{X} \eta\right)(Y)=h \eta(X) \eta(\phi Y)=0$ since $P$ is almost contact. Since $\phi$ has vanishing covariant derivative it is easily seen that $P$ is normal. Indeed, it is easily checked that (2.5) is satisfied for a basis of coordinate vector fields compatible with $\phi$. Hence, $P$ is cosymplectic. Observe that $h=H(\xi, \xi)$.

Conversely, if the almost contact structure on $P$ is cosymplectic, 
$D \eta$ vanishes (see [1]). Consequently, by (3.18), $H(X, \phi Y)=0, X$, $Y \in E(P)$. Hence, $H\left(X, \phi^{2} Y\right)=0$ implies $H(X, Y)=\eta(Y) H(X, \xi)$. But $H(X, \xi)=\eta(X) H(\xi, \xi)$, so

$$
H(X, Y)=H(\xi, \xi) \eta(X) \eta(Y) \text {. }
$$

REMARKS. (a) A simply connected totally geodesic orientable hypersurface of a Kaehler manifold cannot be compact, since otherwise its first betti number is not zero by virtue of the fact that $\eta$ is harmonic.

(b) Observe that the vector field $\xi$ of Theorem 1 is a characteristic vector of $H$.

Proposition 2. A sufficient condition that a $C^{\infty}$ orientable hypersurface $P$ of a Kaehler manifold $M$ be a contact metric manifold is that.

$$
H=\lambda(,)+\mu \eta \otimes \eta, \quad \lambda \neq 0
$$

where (, ) is the almost contact metric and $\eta$ the almost contact. form of $P$.

$$
\begin{aligned}
& \text { Proof. If } H=\lambda(,)+\mu \eta \otimes \eta, \text { then by (3.18), } \\
& \begin{aligned}
2 d \eta(X, Y)= & \left(D_{Y} \eta\right)(X)-\left(D_{X} \eta\right)(Y)=H(Y, \phi X)-H(X, \phi Y) \\
= & \lambda[(Y, \phi X)-(X, \phi Y)]=2 \lambda \Phi(X, Y) .
\end{aligned}
\end{aligned}
$$

Since $M$ is a Kaehler manifold $\Phi$ is closed. Thus, $\lambda$ is a constant and $\Phi=d \eta^{\prime}$ where $\lambda \eta^{\prime}=\eta$.

Observe that $\lambda+\mu=H(\xi, \xi)$.

Theorem 2 is an immediate consequence of the well-known formula

$$
K_{S}(X, Y)=K_{R}(X, Y)-\left[H(X, X) H(Y, Y)-(H(X, Y))^{2}\right]
$$

where $X, Y \in P_{m}$ is an orthonormal pair. For, $K_{S}=0$ and $H(X, Y)=$ $h \eta(X) \eta(Y)$.

Proof of Theorem 3. Let $P_{1}$ and $P_{2}$ be almost contact manifolds with almost contact structures $\left(\phi_{i}, \xi_{i}, \eta_{i}\right), i=1,2$, respectively. Then, an almost complex structure $J$ is induced on the product manifold $P_{1} \times P_{2}$ (see [4]). In fact, for $x_{i} \in P_{i m_{i}}, i=1,2$ we set

$$
J_{\left(m_{1}, m_{2}\right)}\left(x_{1}, x_{2}\right)=\left(\phi_{1} x_{1}-\eta_{2}\left(x_{2}\right) \xi_{1}, \phi_{2} x_{2}+\eta_{1}\left(x_{1}\right) \xi_{2}\right) \text {. }
$$

Now, for $i=1,2$, let $P_{i}$ be given a cosympletic structure with underlying almost contact structure $\left(\phi_{i}, \xi_{i}, \eta_{i}\right)$. Since these structures on $P_{1}$ and $P_{2}$ are normal the almost complex structure $J$ defined by (4.1) on the product manifold $M=P_{1} \times P_{2}$ comes from a complex structure. Define a metric $g$ on $M$ by $g_{1}+g_{2}$ where $g_{i}(i=1,2)$ is the almost contact metric of $P_{i}$. Defining a 2 -form $\Omega$ on $M$ by 


$$
\Omega=\Phi_{1}+\Phi_{2}+\eta_{1} \wedge \eta_{2}
$$

we see that $\Omega$ has maximal rank since $\Phi_{i}$ has this property on $P_{i}$, $i=1,2$. Moreover, since $\Phi_{1}, \Phi_{2}, \eta_{1}$ and $\eta_{2}$ are closed, so is $\Omega$. It is not difficult to check that

$$
g(X, Y)=\Omega(X, J Y),
$$

so $\Omega$ is the Kaehler form of the Kaehler manifold $(M, J, g)$.

\section{REFERENCES}

1. D. E. Blair, The theory of quasi-Sasakian structures, J. of Diff. Geom. 1 (1967), 331-345.

2. D. E. Blair and S. I. Goldberg, Topology of almost contact manifolds J. of Diff. Geom. 1 (1967), 347-354.

3. P. Libermann, Sur les automorphismes infinitésimaux des structures symplectiques et des structures de contact, Coll. de géom. diff. globale, CBRM (1959), 37-59.

4. A. Morimoto, On normal almost contact structures, J. Math. Soc. Japan 15 (1963), 420-435.

5. M. Okumura, Cosymplectic hypersurfaces in Kaehlerian manifold of constant holomorphic sectional curvature, Kōdai Math. Sem. Rep. 17 (1965), 63-73.

6. Y. Tashiro, On contact structure of hypersurfaces in complex manifolds, I, Tôhoku Math. J. 15 (1963), 62-78.

Received December 20, 1967. Research supported by NSF Grant GP-5477.

UNIVERSITY OF ILLINOIS, URBANA 



\section{PACIFIC JOURNAL OF MATHEMATICS}

\section{EDITORS}

\section{H. ROYDEN}

Stanford University

Stanford, California

\author{
R. R. Phelps \\ University of Washington \\ Seattle, Washington 98105
}

\section{J. DugundJI}

Department of Mathematics University of Southern California Los Angeles, California 90007

\section{RICHARD ARENS}

University of California

Los Angeles, California 90024

\section{ASSOCIATE EDITORS}

\section{E. F. BECKENBACH}

B. H. NEUMANN

F. WOLF

K. YOSIDA

\section{SUPPORTING INSTITUTIONS}

UNIVERSITY OF BRITISH COLUMBIA CALIFORNIA INSTITUTE OF TECHNOLOGY UNIVERSITY OF CALIFORNIA MONTANA STATE UNIVERSITY UNIVERSITY OF NEVADA NEW MEXICO STATE UNIVERSITY OREGON STATE UNIVERSITY UNIVERSITY OF OREGON OSAKA UNIVERSITY UNIVERSITY OF SOUTHERN CALIFORNIA

\author{
STANFORD UNIVERSITY \\ UNIVERSITY OF TOKYO \\ UNIVERSITY OF UTAH \\ WASHINGTON STATE UNIVERSITY \\ UNIVERSITY OF WASHINGTON \\ AMERICAN MATHEMATICAL SOCIETY \\ CHEVRON RESEARCH CORPORATION \\ TRW SYSTEMS
}

NAVAL WEAPONS CENTER

Mathematical papers intended for publication in the Pacific Journal of Mathematics should be in typed form or offset-reproduced, double spaced with large margins. Underline Greek letters in red, German in green, and script in blue. The first paragraph or two must be capable of being used separately as a synopsis of the entire paper. It should not contain references to the bibliography. Manuscripts, in duplicate if possible, may be sent to any one of the four editors. All other communications to the editors should be addressed to the managing editor, Richard Arens, University of California, Los Angeles, California 90024.

Each author of each article receives 50 reprints free of charge; additional copies may be obtained at cost in multiples of 50 .

The Pacific Journal of Mathematics is published monthly. Effective with Volume 16 the price per volume (3 numbers) is $\$ 8.00$; single issues, $\$ 3.00$. Special price for current issues to individual faculty members of supporting institutions and to individual members of the American Mathematical Society: $\$ 4.00$ per volume; single issues $\$ 1.50$. Back numbers are available.

Subscriptions, orders for back numbers, and changes of address should be sent to Pacific Journal of Mathematics, 103 Highland Boulevard, Berkeley 8, California.

Printed at Kokusai Bunken Insatsusha (International Academic Printing Co., Ltd.), 7-17, Fujimi 2-chome, Chiyoda-ku, Tokyo, Japan.

PUBLISHED BY PACIFIC JOURNAL OF MATHEMATICS, A NON-PROFIT CORPORATION

The Supporting Institutions listed above contribute to the cost of publication of this Journal, but they are not owners of publishers and have no responsibility for its content or policies. 


\section{Pacific Journal of Mathematics}

\section{Vol. 27, No. $2 \quad$ February, 1968}

Leonard E. Baum and George Roger Sell, Growth transformations for

functions on manifolds ............................ 211

Henry Gilbert Bray, A note on CLT groups ................... 229

Paul Robert Chernoff, Richard Anthony Rasala and William Charles

Waterhouse, The Stone-Weierstrass theorem for valuable fields....... 233

Douglas Napier Clark, On matrices associated with generalized

interpolation problems ................................

Richard Brian Darst and Euline Irwin Green, On a Radon-Nikodym theorem for finitely additive set functions . ...................... 255

Carl Louis DeVito, A note on Eberlein's theorem..................... 261

P. H. Doyle, III and John Gilbert Hocking, Proving that wild cells exist . . . 265

Leslie C. Glaser, Uncountably many almost polyhedral wild $(k-2)$-cells in

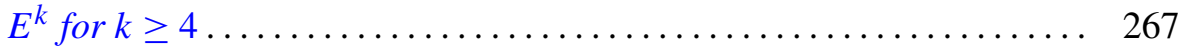

Samuel Irving Goldberg, Totally geodesic hypersurfaces of Kaehler

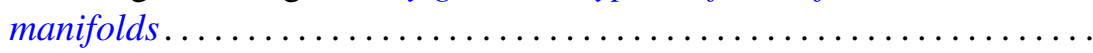

Donald Goldsmith, On the multiplicative properties of arithmetic functions .................................... 283

Jack D. Gray, Local analytic extensions of the resolvent ............ 305

Eugene Carlyle Johnsen, David Lewis Outcalt and Adil Mohamed Yaqub,

Commutativity theorems for nonassociative rings with a finite division ring homomorphic image ....................

André (Piotrowsky) De Korvin, Normal expectations in von Neumann

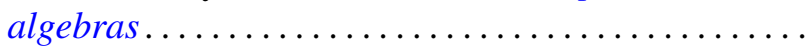

James Donald Kuelbs, A linear transformation theorem for analytic

Feynman integrals..........................

W. Kuich, Quasi-block-stochastic matrices ................... 353

Richard G. Levin, On commutative, nonpotent archimedean

semigroups ............................... 365

James R. McLaughlin, Functions represented by Rademacher series ... . . . 373

Calvin R. Putnam, Singular integrals and positive kernels............ 379

Harold G. Rutherford, II, Characterizing primes in some noncommutative

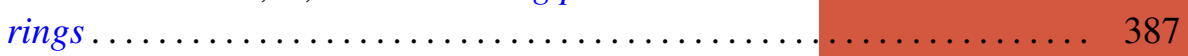

Benjamin L. Schwartz, On interchange graphs................... 393

Satish Shirali, On the Jordan structure of complex Banach *algebras . . . . . 397

Earl J. Taft, A counter-example to a fixed point conjecture............. 405

J. Roger Teller, On abelian pseudo lattice ordered groups ..... 